\title{
SWATH-MS-facilitated proteomic profiling of fruit skin between Fuji apple and a red skin bud sport mutant
}

\author{
Mo-Xian Chen ${ }^{1 \dagger}$, Chao Sun ${ }^{2 \dagger}$, Kai-Lu Zhang ${ }^{1}$, Yu-Chen Song ${ }^{1}$, Yuan Tian', Xi Chen ${ }^{3}$, Ying-Gao Liư ${ }^{4}$ Neng-Hui Ye ${ }^{5}$, \\ Jianhua Zhang ${ }^{6}$, Shenchun $\mathrm{Qu}^{2^{*}}$ and Fu-Yuan Zhu ${ }^{1 *}$ (i)
}

\begin{abstract}
Background: Apple is one of the most popular fruit crops world-wide and its skin color is an important quality consideration essential for commercial value. However, the strategy on genetic breeding for red skin apple and the genetic basis of skin color differentiation is very limited and still largely unknown.

Results: Here, we reported a bud sport mutant of Fuji apple with red skin color and enhanced anthocyanins accumulation. Quantitative SWATH-MS (sequential window acquisition of all theoretical spectra-mass spectrometry) proteomics investigations revealed proteome changes in the apple red skin bud mutation and a total of 451 differentially expressed proteins were identified in apple skin. The mutant showed significantly increased expression levels of photosynthesis-related proteins, stress-related proteins as well as anthocyanins biosynthesis pathway. On the other hand, substantial downregulation of mitogen-activated protein kinase 4 (MAPK4) and mevalonate kinase (MVK) were detected, indicating a promising role for the red skin color development in the mutant. Furthermore, we also hypothesize that a post-transcriptional regulation of the skin color formation occurs in the mutant through the advanced SWATH-MS analysis.

Conclusion: Our work provides important information on the application of proteomic methods for analysing proteomes changes in Fuji apple and highlights a clade of regulatory proteins potentially contributing for the molecular breeding of fruit skin color.
\end{abstract}

Keywords: Bud sport, Skin color, SWATH-MS, Post-transcriptional regulation

\section{Background}

Apple (Malus domestica) is one of the most economically important fruit crops world-wide and its demand from consumers continuously grow based on the nutritional value and broad health benefits. Apple quality mainly depends on multiple characteristics including size, taste, sweetness, texture and bright skin-color $[1,2]$. Interestingly, we often use red skin-color as phenotypic outward assessment for judging the quality of apples. Actually,

\footnotetext{
* Correspondence: ascnj@njau.edu.cn; fyzhu@njfu.edu.cn

${ }^{\dagger}$ Mo-Xian Chen and Chao Sun contributed equally to this work.

${ }^{2}$ College of Horticulture, Nanjing Agricultural University, No. 1 Weigang, Nanjing 8210095, China

${ }^{1}$ Co-Innovation Center for Sustainable Forestry in Southern China, College of Biology and the Environment, Nanjing Forestry University, Nanjing 210037, Jiangsu Province, China

Full list of author information is available at the end of the article
}

optimize any one of them would bring quality improvement in apple cultivation.

Apple fruit coloration is considered as an apparent indicator of maturity and outer quality during the fruit breeding [3-5]. Basically, a group of natural flavonoid compounds especially anthocyanins are essential for fruit coloration, accounting for $80 \%$ of total skin pigmentation in apple [6]. And most of genes including phenylalanine ammonia-lyase (PAL), chalcone isomerase (CHI), dihydroflavonol4-reductase (DFR) involving the anthocyanins biosynthetic pathway have been also well characterized in apple [7-9]. The anthocyanins accumulated in the red skin have the antioxidative and hepatoprotective effects contributing to the health benefits of fruits $[10,11]$. However, besides anthocyanins, various unknown genetic and environmental factors have

(C) The Author(s). 2019 Open Access This article is distributed under the terms of the Creative Commons Attribution 4.0 International License (http://creativecommons.org/licenses/by/4.0/), which permits unrestricted use, distribution, and 
markedly influenced on the skin colour of apple. Few studies have been carried out to investigate the underlying molecular components on the comparisons between different cultivars and mutation. The Asian apple cultivar "Fuji" was commercialized in China whereas a red bud mutation of "Fuji" apple showing red skin pigmentation was recently discovered in Jiangsu Province, China. This bud mutation apple is always red spanning the entire skin-coloration development stage with stable phenotype through continuous observation and grafting experiment. Therefore, to dissect the potential molecular mechanisms of skin-coloration from this mutant and identify the candidate genes controlling the formation of red color is very meaningful for the molecular breeding of apple cultivation.

Transcriptomic and metabolomics analyses on apple fruit color have been investigated to provide insights on the global mRNA profiling and pigmentation metabolites in the formation of apple skin color $[6,12]$. However, genes expression regulation may not adequately reflect the corresponding protein expression due to the various posttranscriptional modifications [13]. So far, understanding on the regulatory mechanisms of apple fruit coloration and mutation at proteome levels remains unclear due to the few proteomic studies reported. In this study, changes in the proteome profile between the "Fuji" apple and a red bud mutation were determined by the SWATH-MS (sequential windowed acquisition of all theoretical mass spectra) approach which has been extensively applied into research model plant such as Arabidopsis and rice $[14,15]$. SWATH-MS experiment perform on the data-independent acquistion (DIA) mode, which combines the advantages of high-throughput of shotgun proteomics and high reproducibility of SRM proteomics, leading to the large-scale protein identification and protein quantification accuracy [16]. It has received considerable attention in plant proteome research due to the higher accuracy and reproducibility in comparison to the conventional approach such as 2D-gel (Two-dimensional gel electrophoresis) and iTRAQ. In present work, 1470 unique proteins were quantitatively identified in "Fuji" apple skin. Amongst them, a total of 451 differentially expressed proteins were observed during the fruit development between "Fuji" apple and its bud mutation. Go and pathway enrichment analysis revealed substantial proteome changes distributing on these biological aspects including photosynthesis and energy metabolism, stress related proteins as well as anthocyanins biosynthesis pathway. Following bioinformatics data analysis would offer considerable information on proteome differentiates between the "Fuji" apple and its bud mutation, acquiring comprehensive understanding on the molecular mechanisms of skin-color development in apple.

Taken together, our SWATH-MS based proteomic investigation may reveal their genetic differences of apple skin-color formation at the protein levels and determine crucial modulator for potential application to the high quality and large fruit-shaped as well as bright color apple cultivars in future.

\section{Result \\ SWATH-MS quantitative proteomics analysis in the Fuji red skin color mutant (Fuji_M)}

Fuji apple (Malus $x$ domestica Brokh cv. Fuji) and the red skin color mutant Fuji_M were found in Shilaojia, Jiangsu Province during the harvest season of 2017. Their young fruits were grown for 30 days after pollination and then were covered with brown bags. The differentiate color of fruits were photographed at 16 day after bag removal as shown in Fig. 1a. It was well known anthocyanins have been identified as an important indicator responsible for fruit skin color formation in many plants $[3,4]$. Therefore, to ensure an appropriate sampling time point between Fuji_apple and Fuji_M for proteomic analyses, the endogenous anthocyanin contents were determined by LC-MS/MS. After bag removal, the anthocyanin levels increased gradually during the fruit maturation but the accelerated rate in the Fuji_ $\mathrm{M}$ were higher than Fuji_apple (Fig. 1b). The maximum differences of anthocyanin contents occurred at 16 days after bag removal for Fuji_apple and Fuji_M (Fig. 1b). Therefore, the 16 DABR (fruits were exposed to normal sunshine after bag removed) were chosen for this proteome study to investigate the proteome changes between Fuji_apple and Fuji_M during the fruit maturation by the SWATH-MS analysis. After merging the data from six biological replicates, approximately 1470 unique proteins were identified and quantified with critical false discovery rate of $1 \%$ (Additional file 1: Table S1). Proteins with a fold change of above 1.5 or below $0.67(P<$ $0.05)$ were considered as differentially expressed proteins (DEPs) in this study. Accordingly, in all of 451 proteins showing significant protein abundance changes between Fuji_apple and Fuji_M with 135 increased and 316 decreased (Table 1). A volcano picture of differentially expressed proteins also revealed numbers of DEPs showing increased abundances in red skin color mutant were 2-3 folds than in the Fuji_apple (Fig. 2a), indicating some crucial regulators or pathways were induced potentially contributing for the red skin phenotype.

Subsequently, functional classification of those DEPs was performed by the Gene Ontology (GO) analysis including Biological Process, Cellular Component and Molecular Function. Accordingly, proteins belonging to the categories "Oxidation-reduction process" and 


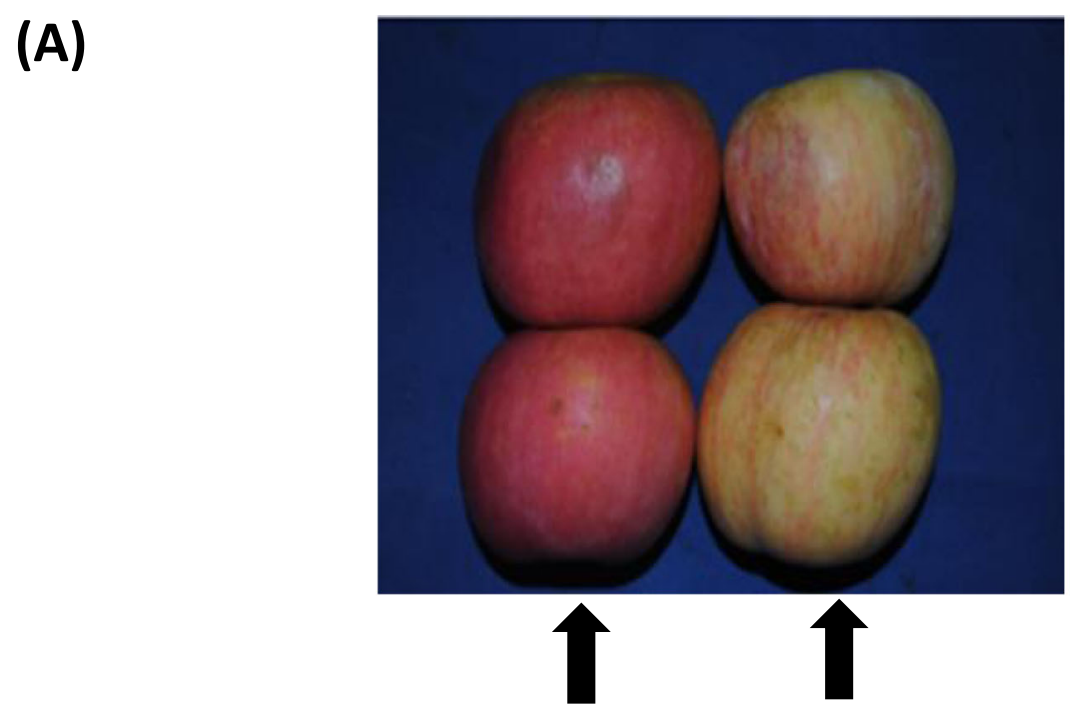

\section{Fuji_M Fuji_Apple}

(B)

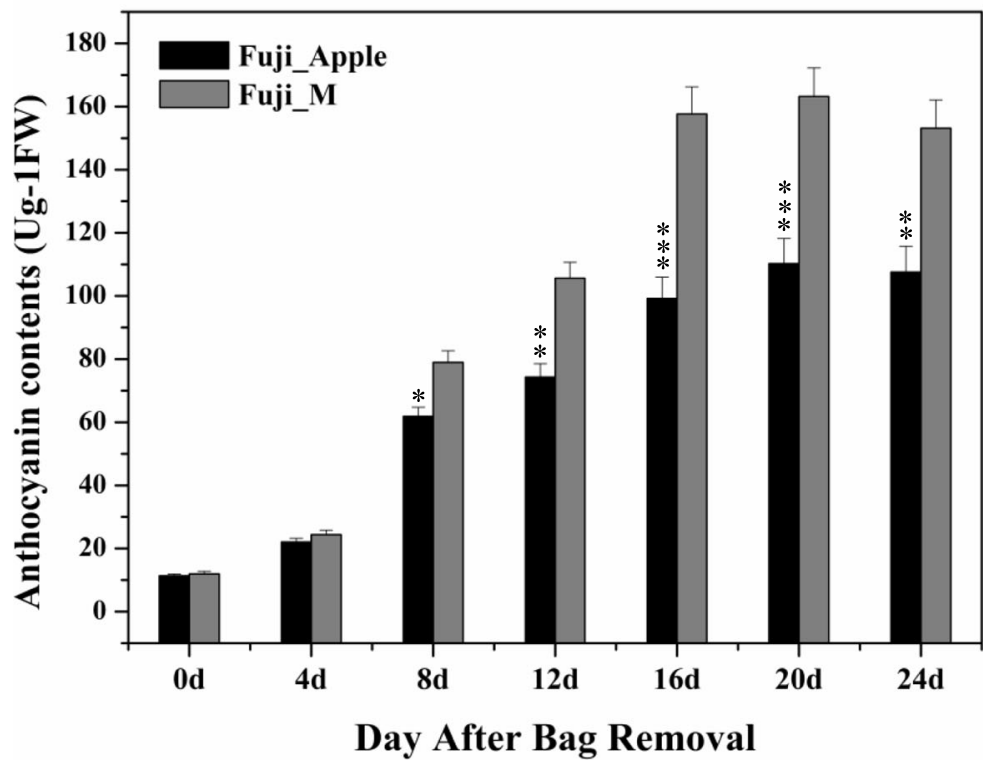

Fig. 1 Phenotypes of "Fuji" apple and its red bud mutation (Fuji_M). a. Representative phenotype was photographed at 16 day after bag removal. b Anthocyanin contents measurement for sampling. Data are mean of five replicates per treatment. ${ }^{*} P<0.05,{ }^{* *} P<0.01,{ }^{* *} P<0.001$ by Student's $t$ test

"Carbohydrate metabolic process" contain the largest number of DEPs, indicating that these courses were greatly affected in the red skin bud mutation (Fig. 2b). It is also worth noting that proteins possessing oxidoreductase activity and magnesium activity were highly represented in the GO term of "Molecular Function", indicating these enzymes were co-ordinately regulated for the red skin color formation. The majority of the DEPs assigned with the GO term of "Cellular Component" were distributed in ribosome and cytoplasm (Fig. 2b). Therefore, these findings from GO analysis indicated that different corresponding biological 
Table 1 Distribution of DEPs numbers

\begin{tabular}{ll}
\hline CK VS M & Number of unique proteins \\
\hline Increased $(F C \geq 1.5, P<0.05)$ & 135 \\
Decreased $(F C \leq 0.67, P<0.05)$ & 316 \\
Total & 451 \\
\hline
\end{tabular}

processes or enzymes were activated or repressed in red skin bud mutation to fulfil blushed properties in apple. Moreover, KEGG (Kyoto Encyclopedia of Genes and Genomes) pathway classifications were also conducted on the DEPs offering a quick view to the most significant pathway in the bud mutation (Fig. 3). We listed top 20 of pathway such as glycolysis, carbon fixation in photosynthetic process, amino acid metabolism upon the mapping of DEPs (Fig. 3), among which should be the key for the differentiate phenotype in apple.

\section{Photosynthetic and energy metabolism proteins}

The extent of photosynthesis is critical for the fruit skin color changes. The fruit color from green to red always depend on the reduction or suppression of chlorophyll content to appear the color of anthocyanins by the regulation of photosynthesis proteins $[17,18]$. In this study, there are 18 photosynthesis-related proteins identified with altered abundances. Interestingly, most of all were up-regulated in the red skin mutant, among which are important elements of photosynthetic enzyme complexes including cytochrome b6/f, photosystem I and II (PSI and PSII) and light-harvesting chlorophyll protein complex (LHC) (Additional file 2: Table S2), strongly suggesting that the light reactions were reinforced in the red skin color mutant compared with Fuji_apple. Similarly, $\mathrm{CO}_{2}$ assimilation was also shown to be accelerated with increased protein abundances of Calvin cycle enzymes in the mutant, including rubiscoactivase (MDP0000944409) required for $\mathrm{CO}_{2}$ fixation and glyceraldehyde-3-phosphate dehydrogenase (MDP0000 835914, MDP0000527995) involved in the reduction stage as well as D-ribulose-5-phosphate-3-epimerase (MDP0000276466) involving the regeneration of ribulose bisphosphate. Interestingly, NAD-dependent malate dehydrogenase (MDH) was also found to show increased abundances in the mutant, which is a key enzyme catalysing hydrogen ion of hydroxyl in malice acid for oxaloacetate regeneration, indicating the enhancement of respiratory metabolism in the red skin bud mutation. Therefore, the overall up-regulation of photosynthetic activities in this mutant probably due to the demand of light absorption by the accumulation of anthocyanins, resulting in the improvement of the ability of light reaction, carbon assimilation as well as respiration for the red skin bud mutation.

\section{Upregulation of anthocyanins biosynthetic pathway in Fuji red skin color mutant}

Anthocyanins are essential components in red coloration sources for apple skin color [5]. The red-skin cultivars accumulate more anthocyanins than the pale or white ones, which have been considered as an important fruit trait influencing the market values. Here, several key enzymes with enhanced abundance changes were participated and mapped into sequential reactions of anthocyanins biosynthesis (Fig. 4a). Anthocyanins are synthesized from coumaroyl-CoA and malonyl-CoA by the catalysation of chalcone synthase (CHS, MDP0000287919) to form chalcones. Afterward, chalcone isomerase (CHI, MDP0 00013791) catalyses the conversion of chalcones to flavanones. The next step is catalysed by a flavanone-3ßhydroxylase $(\mathrm{F} 3 \mathrm{H})$ resulting in the formation of dihydroflavonols. On the one hand, dihydroflavonols is then transferred for reduction bydihydroflavonol-4-reductase (DFR, MDP000484976) to form leucoanyhocyanidins. On the other hand, dihydroflavonols are also entered into the production of flavonols by flavonol synthase (FLS, MDP0000183682) through sequential reactions. The conversion of leucoanyhocyanidins to cyanidins-based anthocyanins was finished by final two enzymes including LDOX and UFGT, respectively. Therefore, those clades of enzymes with increased abundances changes directly reflect the upregulation of anthocyanins biosynthesis in Fuji_M (Fig. 4a), which also consistent with results from the anthocyanins contents determination between Fuji Apple and Fuji_M (Fig. 1b). Furthermore, we investigated the anthocyanins compositions by the LC-MS/MS analysis and observed many types of anthocyanins contained in apple skin. Particularly, the cyanidin-3-galactoside (cy-3gal) detected in the Fuji_M was significantly increased by approximately $50 \%$ in comparison to the Fuji_Apple (Fig. $4 \mathrm{~b})$, which was also the most abundant anthocyanin in apple [6]. Therefore, the significant skin-color differences in Fuji_Apple and Fuji_M are probably due to the regulation of anthocyanins biosynthesis. Interestingly, this kind of regulatory mechanism is mainly based on a transcription complex, which consist of three transcription factors belonging to the MYB, bHLH and WD40 classes [4, 17], activating anthocyanin biosynthesis pathway by the MYBbHLH-WD40 complex in most plants [19]. However, no MYB and bHLH-related proteins were found and only two WD40 repeat-like proteins (MDP0000231283 and MDP0000433881) with enhanced abundances in the mutant were quantitatively identified from this study (Additional file 2: Table S2). Several reports revealed WDrepeat proteins promotes the accumulation of anthocyanins in plants including apple, green tea and tartary 


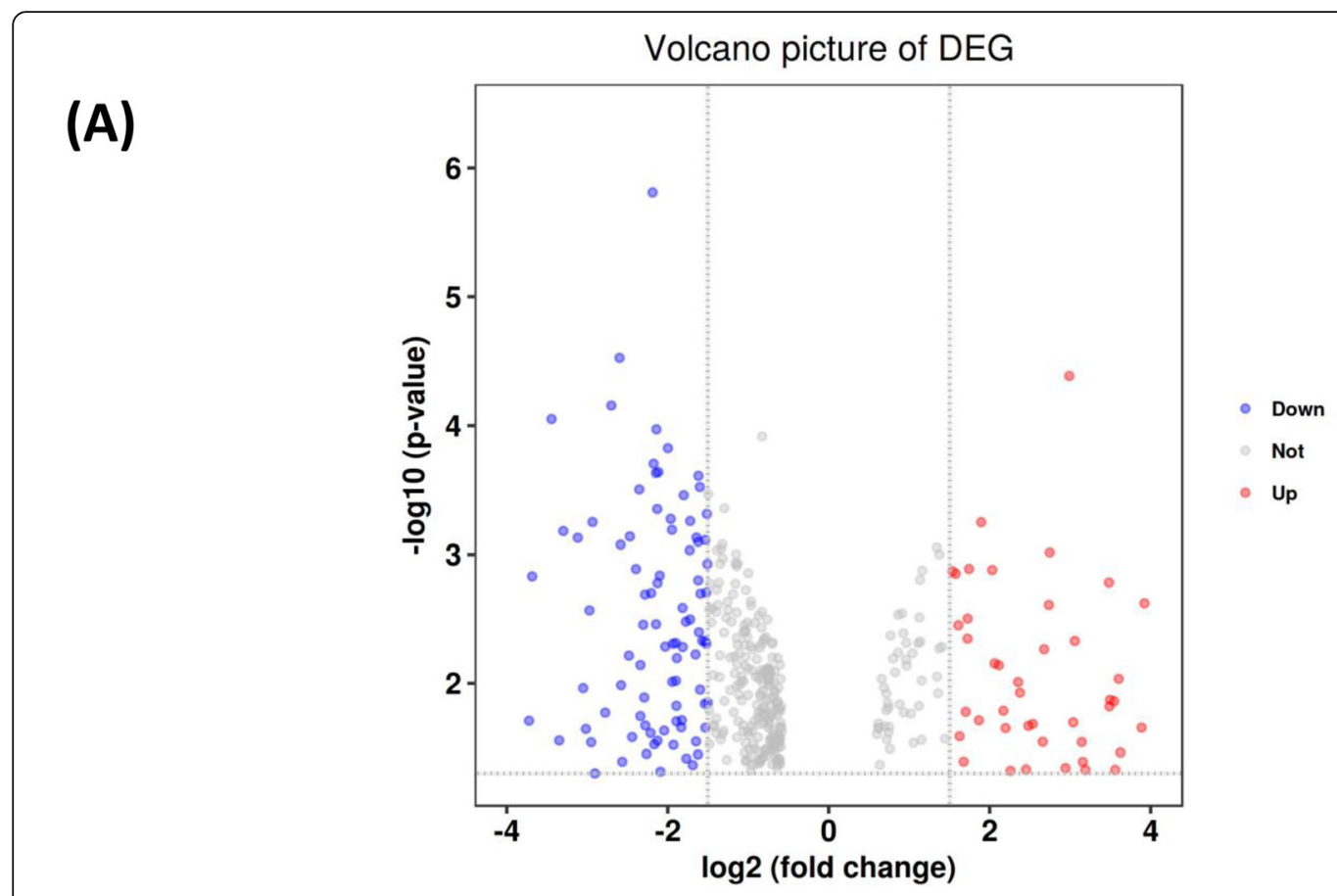

(B)

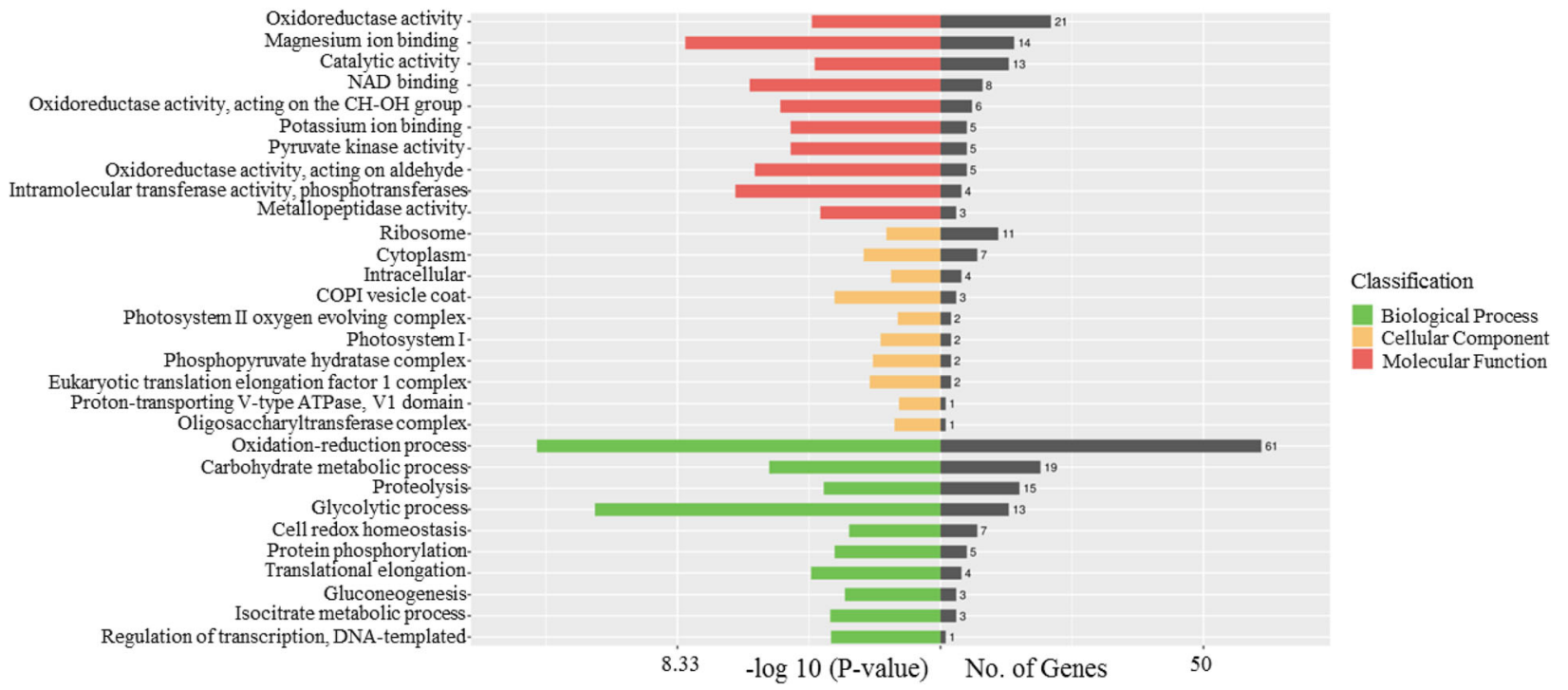

Fig. 2 Functional categorization of the differentially expressed proteins by the Gene Ontology (GO) analysis. a The volcano picture showing the distribution of the differentially expressed proteins with different fold changes. $\mathbf{b}$ The plots reveal the differentially expressed proteins distribution based on three ontologies including Biological Process, Cellular Component and Molecular Function

buckwheat [20-22]. The apple WD40 protein regulate anthocyanin biosynthesis through the interaction of bHLH rather than the activation of MYB-bHLH-WD40 complex [23]. Therefore, the upregulation of two
WD40 classes proteins caused by the disinhibition of potential mutated gene probably regulate the anthocyanins accumulation in Fuji_M, thus contributing to the red skin coloration. 


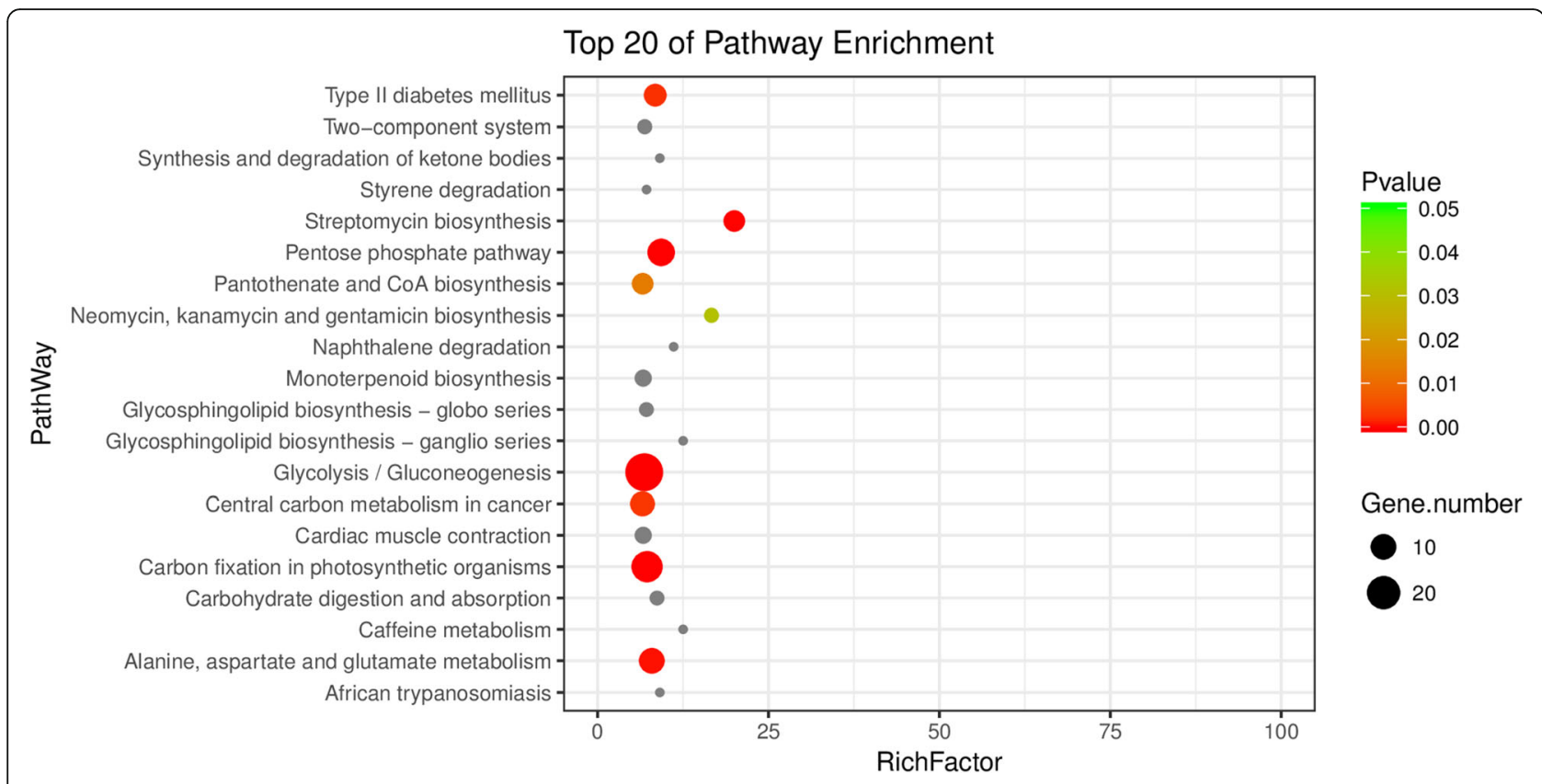

Fig. 3 KEGG classifications on the DEPs. RichFactor represents the degree of enrichment

\section{Stress related proteins}

In our study, some identified differentially expressed proteins showed than increased abundances in Fuji_M were enriched in the component of stress-related proteins and they are likely to be participated in various biotic and abiotic stresses (Additional file 2: Table S2). It was well known that stress-related proteins are spontaneously synthesized for the plant resistances coping with environmental stress factors including abnormal temperature, heavy metals, drought and salinity [24, 25]. Here, several heat shock proteins (HSPs: HSP70; HSP81.4; HSP90.1; HSP101) were detected to be accumulated in the Fuji-M (Additional file 2: Table S2), which is one kind of conservative stress family proteins. For example, HSP70 is able to involving the disposal of damaged or defective proteins to proteolysis pathway through its molecular chaperone function with E2 ubiquitin ligase [26, 27]. Coincidently, we also found unique E2 (MDP0000714492) with significant increasing abundances in this red skin mutant (Fuji-M), suggesting that this kind of cellular protective machinery was conserved and essential for the red skin bud mutation (Fuji-M). Therefore, the red skin mutant probably possesses improved resistance ability compared to the Fuji_Apple, which will need further investigation. Interestingly, Hsp70 also has been reported to participate in human retinal pigment epithelium through its chaperone function with a linkage of proteolysis pathway [28], further indicating HSP70 may be involved in the apple skin pigmentation process. In addition, three S-adenosyl-L-methionine-dependent methyltransferases (SAMMTases:
MDP0000277077; MDP0000268065; MDP0000776572) were also upregulated in the Fuji-M. SAMMTases basically function to transfer methyl group from the donor of $\mathrm{S}$-adenosyl methionine (SAM) to various biomolecules, playing critical roles in biosynthetic pathways of major natural products including lipids, terpenoids, nucleic acids and flavonoids, particularly in their biological activities through the modulation of the methylation patterns [29]. Therefore, SAMMTases have considerable efforts on the biosynthesis of flavonoids in the apple, which are highly correlated with skin colour formation. Upregulation of SAMMTases in the Fuji_M may alter the flavonoids structural properties and flavonoids pigmentation leading to the feature of red skin.

\section{Discussion}

Candidate proteins responsible for the red skin mutation

Seeking target proteins for the red skin mutation in the differential expressed proteins between Fuji_Apple and Fuji_M are necessary to illuminate the molecular mechanism of this bud mutation. Here, we collected 16 candidate proteins with dramatic decreasing abundances (Fold changes $>100$ ) in the Fuji_M as shown in Table 2. Representatively, mitogen-activated protein kinase 4 (MAPK4: MDP0000321746) exhibited 116 folds of down-regulation in the Fuji_M. MAPKs play crucial roles in the regulation of cellular signal transduction or as signalling molecules, which have been reported to participate in pigment cell development [30]. Interestingly, flavonoids have inhibitory effects on MAPK activation [31]. Therefore, disruption of MAPK4 and 
(A)

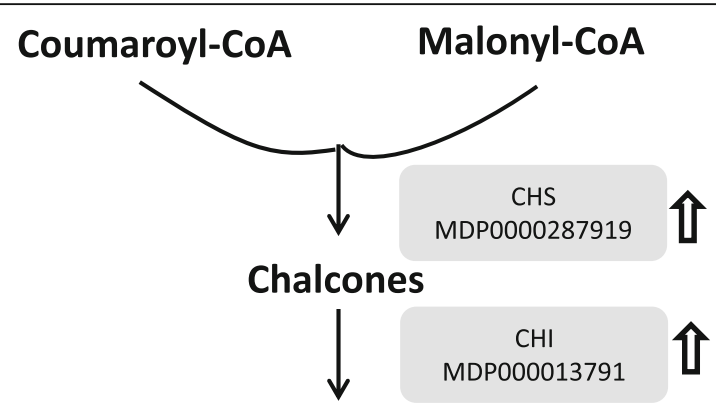

Flavanones

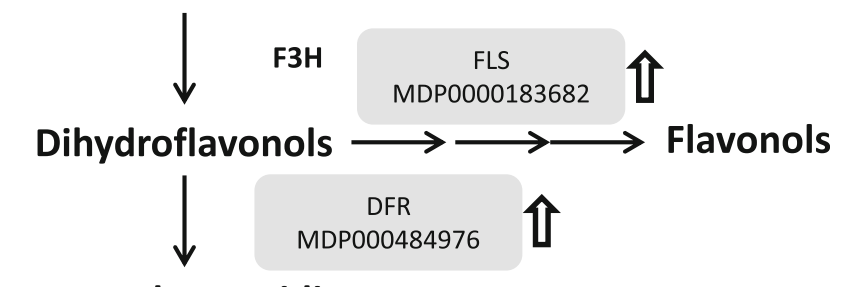

\section{Leucoanyhocyanidins}

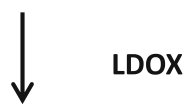

Cyanidins

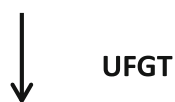

(B)

\section{Cyanidins-3-galactoside}

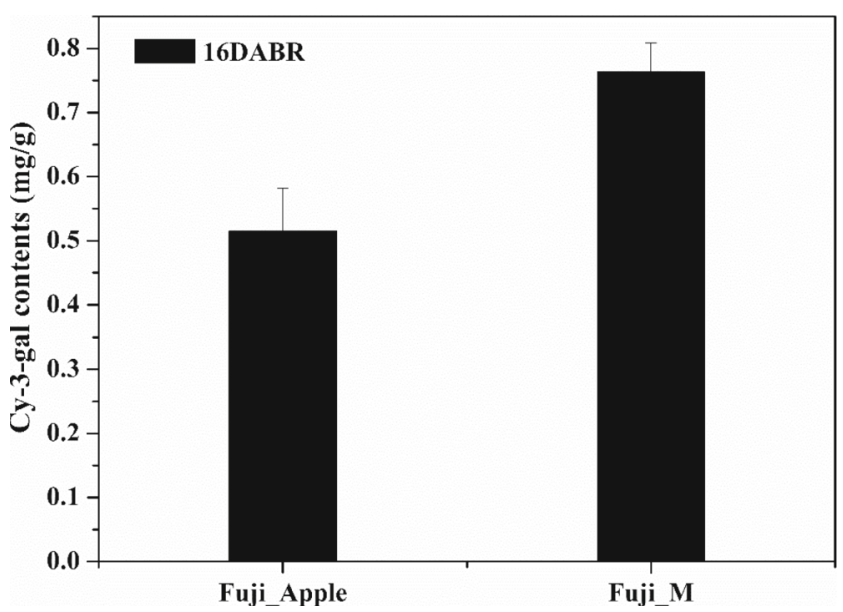

Fig. 4 Anthocyanin biosynthesis was greatly affected in the bud mutation. a Several enzymes involved in anthocyanin biosynthesis showing significant protein abundances changes were mapped to the anthocyanin metabolism pathway. indicates up-regulation. Enzyme abbreviations: CHS, chalcone synthase; $\mathrm{CHI}$, chalcone isomerase; F3H, flavanone-3ß-hydroxylase; DFR, dihydroflavonol-4-reductase; FLS, flavonol synthase. b Cy-3-gal was detected in the Fuji_M and Fuji_Apple at 16 day after bag removal (DABR)

upregulation of flavonoids biosynthesis pathway were observed in the Fuji_M, strongly suggesting that MAPK signalling pathway probably function as a modulator in the apple skin pigmentation process. More interestingly, stress-related protein HSP70 also involved in the MAPK signalling pathway [32], which may serve as a downstream signalling molecule contributing to the red colour formation in apple. On the other hand, another candidate protein mevalonate kinase (MVK: MDP000 0025413) was shown to be greatly down-regulated in the Fuji_M, which is a key enzyme in isoprenoid synthesis. Interestingly, plants use two distinct pathway including 
Table 2 Summary of the candidate proteins responsible for the red skin mutation

\begin{tabular}{llll}
\hline Protein ID & Homolog-Arabidopsis & Description & Fold change $(P<0.05)$ \\
\hline MDP0000128052 & AT1G56340.2 & calreticulin 1a & 112.7 \\
MDP0000327254 & AT1G71950.1 & Proteinase inhibitor, propeptide & 105.7 \\
MDP0000211549 & AT3G47590.1 & alpha/beta-Hydrolases superfamily protein; & 109.5 \\
MDP0000198482 & AT1G79530.1 & chloroplast/plastid localized GAPDH isoforms & 111.1 \\
MDP0000202291 & AT1G02560.1 & nuclear encoded CLP protease 5 & 111.5 \\
MDP0000321746 & AT4G01370.1 & MAP kinase 4 & 116.8 \\
MDP0000153587 & AT5G13430.1 & Ubiquinol-cytochrome C reductase iron-sulfur subunit & 181.4 \\
MDP0000222212 & AT5G08380.1 & alpha-galactosidase 1 & 184.0 \\
MDP0000147913 & AT4G10490.1 & 2-oxoglutarate (2OG) and Fe(II)-dependent oxygenase & 208.3 \\
MDP0000812797 & AT2G44350.2 & Citrate synthase family protein & 215.2 \\
MDP0000523595 & AT5G28060.1 & Ribosomal protein S24e family protein & 223.6 \\
MDP0000182890 & AT1G21880.2 & lysm domain GPl-anchored protein 1 precursor & 227.6 \\
MDP0000225450 & AT1G76690.1 & 12-oxophytodienoate reductase 2 & 237.7 \\
MDP0000470429 & AT1G07920.1 & GTP binding Elongation factor Tu family protein & 298.1 \\
MDP0000025413 & AT5G27450.2 & mevalonate kinase activity & 670.2 \\
MDP0000624197 & AT5G47030.1 & ATPase, F1 complex, delta/epsilon subunit & 1177.7
\end{tabular}

the MEP (Methylerythritol 4-phosphate) and MVA (Mevalonic acid) pathway to synthesize isoprenoid for the function in photosynthesis, pigment production and hormones biogenesis [30]. MVK belonging to the MVA pathway play as a rate-determining enzyme for the production of isoprenoid [33] and suppression on the MVK probably cause a disordered control of isoprenoid supply, which was needed for essential processes such as photosynthesis and pigmentation. Both of them are critical for the formation of skin colour in apple. Moreover, the lowest expression of candidate protein in the Fuji_M compared to the Fuji_Apple encodes a mitochondrial ATP synthase subunit delta belonging to F1 type-ATPase (MDP0000624197). It is a component of mitochondrial respiratory complexes for plants coping with stress induced oxidative damage to respiratory function $[34,35]$. The severe inhibition of this protein in the mutant probably due to the huge cost of photosynthesis as an offset of the weak respiration in mitochondrial. Taken together, further genetic and molecular strategies with bioinformatics analysis will boost to confirm the roles of these candidate proteins controlling fruit skin pigmentation and validate the target protein responsible for the red skin bud mutation.

Proteomic analysis reveals post-transcriptional changes may contribute to red skin bud mutation in apple

Several transcriptome sequencing experiments have been conducted using different plant species such as Arabidopsis, cucumber, maize and potato [36-39]. However, the protein abundance of identified differentially expressed genes remains unclear. Previous SWATH-MS based studies reported that few transcription factors have been found in the list of differentially expressed proteins [15], supporting the claims that the regulation of transcription factors largely occur in posttranslational level. In present work, twelve proteins were observed to be involved in post-transcriptional regulation through RNA processing (Table 3). Referring to the splicing-related gene (SRG) summarized in SRGD database (http://www.plantgdb.org/SRGD/index.php),5 of these genes are classified into 3 major groups of splicing factors, including proteins are involved in spliceosome composition (small nuclear ribonucleoproteins, snRNP), Poly A binding and regulatory splicing factors (glycine-rich RNA binding protein) (Table 3). Amongst them, some proteins (e.g.MDP0000283985, MDP000 0245145 and MDP320945) are highly homologous with RNA processing related proteins in Arabidopsis, which have been well studied to participate in splicing process such as spliceosome assembly or splicing site selection [40, 41], implying that, other than traditional transcriptional control, substantial changes in alternative splicing may exist as an extra layer of regulatory mechanism during the skin colour formation [42]. Meanwhile, MDP0000260339, a homologue of Arabidopsis glycinerich RNA binding protein (AtGRP7) which participates in development and abiotic stress responses [43-46], was significantly up-regulated in Fuji_M. Interestingly, several isoforms of AtGRP7 proteins were also detected by proteomic analysis to respond environmental stress [47], further suggesting the post-transcriptional regulation presumably exist during apple skin color formation. In 
Table 3 List of RNA processing related proteins in DEPS.

\begin{tabular}{|c|c|c|c|c|c|}
\hline Protein ID & $\begin{array}{l}\text { Homolog- } \\
\text { Arabidopsis }\end{array}$ & Functional classification & Description & $\begin{array}{l}\text { Fold change } \\
\text { (CK VS M) }\end{array}$ & P-Value \\
\hline MDP0000320341 & AT1G09340.1 & RNA & Chloroplast RNA binding & 0.166 & 0.001 \\
\hline MDP0000283985 & AT4G34110.1 & RNA.processing & Poly $(A)$ binding protein 2 & 0.593 & 0.009 \\
\hline MDP0000245145 & AT4G17100.1 & RNA.processing & $\begin{array}{l}\text { Poly(U)-specific endoribonuclease-B } \\
\text { protein }\end{array}$ & 0.129 & 0.029 \\
\hline MDP0000320945 & AT2G18510.1 & RNA.processing.splicing & $\begin{array}{l}\text { RNA-binding (RRM/RBD/RNP motifs) } \\
\text { family protein }\end{array}$ & 0.453 & 0.004 \\
\hline MDP0000272708 & AT2G27040.1 & $\begin{array}{l}\text { RNA.regulation of } \\
\text { transcription.Argonaute }\end{array}$ & Argonaute family protein & 0.268 & 0.005 \\
\hline MDP0000213737 & AT2G17390.1 & $\begin{array}{c}\text { RNA.regulation of } \\
\text { transcription.AtSR Transcription } \\
\text { Factor family } \\
\end{array}$ & Ankyrin repeat-containing $2 \mathrm{~B}$ & 0.659 & 0.016 \\
\hline MDP0000614064 & AT4G27000.1 & $\begin{array}{l}\text { RNA.regulation of } \\
\text { transcription.unclassified }\end{array}$ & $\begin{array}{l}\text { RNA-binding (RRM/RBD/RNP motifs) } \\
\text { family protein }\end{array}$ & 0.269 & 0.020 \\
\hline MDP0000433881 & AT1G18080.1 & $\begin{array}{l}\text { RNA.regulation of } \\
\text { transcription.unclassified }\end{array}$ & $\begin{array}{c}\text { Transducin/WD40 repeat-like superfamily } \\
\text { protein }\end{array}$ & 0.400 & 0.025 \\
\hline MDP0000315228 & AT4G21670.1 & RNA.RNA binding & C-terminal domain phosphatase-like 1 & 0.605 & 0.027 \\
\hline MDP0000260339 & AT2G21660.1 & RNA.RNA binding & Cold, circadian rhythm, and rna binding 2 & 0.496 & 0.004 \\
\hline MDP0000253359 & AT1G56110.1 & $\begin{array}{l}\text { RNA.regulation of } \\
\text { transcription.putative } \\
\text { transcription regulator }\end{array}$ & Homolog of nucleolar protein NOP56 & 3.255 & 0.017 \\
\hline MDP0000232670 & AT3G10640.1 & RNA.regulation of transcription & $\begin{array}{c}\text { SNF7 family protein } \\
\text { VSP60.1(Vacuolar protein sorting- } \\
\text { associated protein } 60.1 \text { ) }\end{array}$ & 33.726 & 0.029 \\
\hline
\end{tabular}

addition, a putative apple heterogeneous ribonucleoprotein (hnRNP), MDP0000614064, was found to be upregulated in Fuji_M. Two homologous proteins (RBP45 and RBP47) has been reported previously in N. plumbaginifolia [48]. These two proteins have been demonstrated to be able to associate with Poly A RNA and was potential components of U1 snRNP [48]. Therefore, the putative interaction between this protein and MDP0000283985, a Poly A binding protein [49] during post-transcriptional regulation was proposed. However, the potential role of MDP0000614064 in the formation of skin color remains elusive. Besides, several RNA-related proteins for the regulation of transcription were also detected in downregulated protein subset (Additional file 2: Table S2), indicating that complex control of RNA stability and transcriptional availability maybe important for plant skin colour development.

\section{Conclusion}

Fruit skin colour formation seems to initiate a complex responsive network, ranging from transcriptional to post-translational control. Thus, traditional experimental design using single transcriptomic or proteomic approach will encounter more difficulties to find true regulator or pathways which are responsible for fruit skin colour. Traditional proteomic methods such as 2D-gel suffer from their instability of protein quantification and large variation [50]. Thus, as a new generation of labelfree method, SWATH-MS based proteomics are believed to enhance the identification of differentially expressed proteins and improve repeatability amongst replicates at the same time $[15,51]$. Besides, shot-gun proteomics are often limited by their reference databases, which can be complemented by self-constructed protein database from parallel short-read RNA sequencing [52]. The development of proteogenomics, a bioinformatic pipeline to integrate data from RNA sequencing and proteomics, become popular in recent years for both animal and plant research [42, 53]. Definitely, functional analysis such as using genetic approaches and constructing transgenic lines is strongly recommended to validate the findings from bioinformatics approach. Although several differential regulation events have been revealed by our investigation, it also possibly appears to be the effect rather than the cause for this red skin bud mutation. Therefore, the integration of proteomics, RNAsequencing and more molecular approaches will elucidate more information in fruit skin colour formation.

The present work offered a global view to understand the molecular differentiation between Fuji apple and 
mutant, which revealed significant protein abundances changes in photosynthetic and energy metabolism, anthocyanin biosynthesis as well as stress responses for the differentiate colour phenotype in apple. Furthermore, several potential target proteins for the red skin mutation such as MAPK4, MVK and F1 type-ATPase were identified with greatly decreased abundances in its color mutant, among which play positively effects on the pigmentation process to the feature of red apple. Additionally, the advanced SWATH-MS analysis also revealed a certain proportion of proteins involving posttranscriptional changes serve as a regulatory role in fruit coloration. Taken together, our proteomic analysis provides new cues for further research in apple skin-color formation and will extend our understanding of skin colouration for future fruit breeding.

\section{Methods}

\section{Plant materials and sampling}

Bud sport are infrequent changes in phenotype particularly affecting flower and fruit color in horticultural plants such as apple and grape [54]. Here, Fuji apple (Malus domestica Brokh cv. Fuji) and the red skin color mutant Fuji_M from its bud sport branch were obtained from the apple orchard under standard orchard practices in city of Shilaojia, Jiangsu province, China. The Fuji apple is a commercially available cultivated variety and collected from Japan. The phenotype of this bud sport mutant has inherently appeared during the grafting and we have acquired the permission of orchard to collect samples and do further research under the national guidelines. Both flowers on normal branches and on the bud spot were pollinated. Young fruits were grown for 30 days after pollination (30 DAP) and then were covered with brown bags (size: $13 \times 16 \mathrm{~cm}$ ). Bags were removed before harvesting (150 DAP) and samples were collected for six time points: $0,4,8,12,16,20,24$ days after bag removal (DABR) for anthocyanin contents analysis and 16 DABR was finally chosen for following proteome study. Each time point was stored and measured with six biological replicates.

\section{Anthocyanin contents analysis}

The measurement is performed according to the previously common methods reported [55, 56]. Briefly, approximately $0.5 \mathrm{~g}$ of apple skin was collected and extracted by the addition of a $10 \mathrm{~mL}$ methanol solution with $1 \%$ hydrochloric acid in the dark at room temperature for $2 \mathrm{~h}$. All samples were measured in five replicates and anthocyanins were determined by spectrophotometer at absorbance of $530 \mathrm{~nm}$ and $600 \mathrm{~nm}$ respectively. The relative anthocyanin content (Q) was calculated by this formula: Q = OD530nm-OD600nm and per 0.01 was defined as an anthocyanin content unit for convenience. Subsequently, different types of anthocyanins in apple were detected by ABI2000-QTrap mass spectrometer (Applied Biosystems). Total plant anthocyanins were extracted according to the method described above. The compositions of anthocyanins in the samples were analysed by HPLC-MS/MS using a ABI2000QTrap and the detailed procedure was as described by [57]. Briefly, extracted and filtered samples $(20 \mu \mathrm{l})$ were trapped on a Nucleosil 100-5 C18 column $(5 \mu \mathrm{m}, 150 \times$ $2 \mathrm{~mm}$, Agilent Technologies) with $95 \%$ solvent A $(0.1 \%$ formic acid in water) and 5\% solvent B (0.1\% formic acid in acetonitrile), followed by separation in a gradient of $15-60 \%$ B over $35 \mathrm{~min}$ at a flow rate of $0.2 \mathrm{~mL} / \mathrm{min}$. The final LC eluted products were determined by an on-line ABI2000-QTrap mass spectrometer (Applied Biosystems) under multiple reaction monitoring (MRM) mode (positive ionization, scan range: $200-1200 \mathrm{~m} / \mathrm{z}$ ).

\section{Protein extraction and peptide preparation}

Approximately $1 \mathrm{~g}$ of apple skin was ground into powder by liquid nitrogen in a mortar. Samples were homogenized with the pre-iced 10X vol of solution A (10\% trichloroacetic acid (TCA)/ acetone) and then centrifuged at $16000 \mathrm{~g}$ for 5 mins. After removed the supernatants, the pellets were washed by the $10 \mathrm{X}$ vol of solution B $\left(80 \% \mathrm{MeOH} / 0.1 \mathrm{M} \mathrm{NH}_{4} \mathrm{OAc}\right)$ and then centrifuged again and followed by the $10 \mathrm{X}$ vol of washing solution $\mathrm{C}$ (Chilled 80\% acetone). By centrifugation with the supernatants removing, the pellets were dissolved into the 8 $\mathrm{ml} \mathrm{SDT}$ buffer (4\% SDS, 0.1 M DTT, $0.1 \mathrm{M} \mathrm{MOPS/Cl}$, $\mathrm{pH}$ 8.0). Thereafter, dissolved sample was incubated at $95^{\circ} \mathrm{C}$ for 10 mins and cooled on ice immediately for 5 mins. After centrifugation twice again, the final supernatants were obtained by $4 \mathrm{X}$ volume of $80 \%$ acetone for protein precipitation overnight at $-20^{\circ} \mathrm{C}$. At the end, the pellets were dissolved into $1-2 \mathrm{ml}$ solution buffer $(6 \mathrm{M}$ urea in $200 \mathrm{mM}$ MOPS-Cl/ $4 \mathrm{mM} \mathrm{CaCl}, \mathrm{pH} 8.0$ ) according to the size of pellet. The protein concentration was determined based on the Bradford method (Bradford Protein Assay, Bio-Rad).

$100 \mu \mathrm{g}$ protein from each sample was reduced by 10 $\mathrm{mM}$ DTT at $50^{\circ} \mathrm{C}$ for 40 mins and then by the alkylation with $40 \mathrm{mM}$ IAA (iodoacetamide) in the dark at room temperature for 30 mins. Subsequently, each sample was further diluted by distilled water for reducing urea concentration less than $2 \mathrm{M}$ and sample peptides were acquired by a trypsin digestion (enzyme/protein, 1 : $50 \mathrm{w} / \mathrm{w}$ ) at $37^{\circ} \mathrm{C}$ overnight. The acquired acidified peptides by $10 \%$ trifluoroacetic acid (TFA) were further desalted by SepPak C18 cartridges (Waters). Moreover, the filtered peptide samples were speed-dried in a vacuum concentrator and directly dissolved into the water solution ( $0.1 \%$ formic acid) for MS analysis. 


\section{SWATH-MS data analysis}

MS analysis were performed by a Triple TOF 5600 mass spectrometer (AB Sciex) coupling with an Eksigent NanoLC-2D plus system. Peptide $(2 \mu \mathrm{g})$ was trapped on a nanoFlexcHiPLC column $(3 \mu \mathrm{m}$, ChromXP C18CL, $120 \AA, 0.5 \mathrm{~mm} \times 200 \mu \mathrm{m}$ ) under the $95 \%$ buffer A (water with $0.1 \%$ formic acid) and $5 \%$ buffer B (acetonitrile with $0.1 \%$ formic acid), followed by a separation through a 120 -min gradient from 5 to $35 \%$ Buffer B at a stable flow rate of $300 \mathrm{~nL} / \mathrm{min}$. Full-scan MS was conducted in a positive ion mode with an ion spray voltage of $2300 \mathrm{~V}$ under the Triple TOF 5600 mass spectrometer. The reference spectral library was firstly constructed by the data-dependent acquisition (DDA) mode. The entire MS1 spectra was gathered by a full-scan of $250 \mathrm{~ms}$ from 350 to $1250(\mathrm{~m} / \mathrm{z})$, among which the top 40 precursor ions were selected for MS/MS fragmentation $(\mathrm{m} / \mathrm{z} 100$ 1800). In the SWATH acquisition mode, the similar chromatographic parameters was used as the DDA mode described above. A set of 50 overlapping windows was created to cover all precursor ions in the range of 400$1250 \mathrm{Da}$ for further MS2 fragmentation through the cyclic DIA (Data-Independent Acquisition) mode. Thereafter, the DDA mass spectrometry files were searched by the software ProteinPilot 4.5 (AB Sciex) against from the Phytozme-Malus domestica v 1.0 protein database (63, 517 protein entries, June 2019; https://phytozome.jgi. doe.gov/pz/portal.html). The parameters including the digestion, alkylation, biological modification and ID search were set up during the database search and a false discovery rate (FDR) of $<1 \%$ was applied into criteria selection for the protein identification and peptides assignments. Subsequently, all DIA raw files and reference library were loaded into the software PeakView v.1.2 (AB Sciex) using restricted parameters such as 8 peptides, 6 transitions, peptide confidence of $>99 \%$ and XIC width $(50 \mathrm{ppm})$. The processed mrkvw files were followed by loading into MarkerView (AB Sciex) for further quantification analysis through the normalization of protein intensity of their peak areas as described in [58, 59]. Protein differential expression between Fuji_Apple and Fuji_M was expressed in ratios as $>1.5$ or $<0.67$ with $p$ value $<0.05$ to be up-regulation or downregulation respectively. All the raw data files have been submitted into the PRIDE PRoteomicsIDEntifications (PRIDE) database with the accession number PXD011132.

\section{Proteomic data analysis}

Gene Ontology (GO) analysis (http://geneontology.org/) and Kyoto Encyclopedia of Genes and Genomes (KEGG; http://www.kegg. jp/) enrichment classification were performed using DEPs data set.

\section{Supplementary information}

Supplementary information accompanies this paper at https://doi.org/10. 1186/s12870-019-2018-1.

Additional file 1: Table S1. Protein reports for the 1470 unique proteins quantified by SWATH-MS. (XLSX 538 kb)

Additional file 2: Table S2. List of differentially expressed proteins following classification analysis. Proteins with fold change $>1.5$ (Increased) or $<0.67$ (Decreased) are considered as DEPs ( $P$ value $<0.05$ ) (XLSX $125 \mathrm{~kb})$

\section{Abbreviations}

CHI: Chalcone isomerase; CHS: Chalcone synthase; DABR: Fruits were exposed to normal sunshine after bag removed; DAP: Days after pollination; DEPs: Differentially expressed proteins; DFR: Dihydroflavonol-4-reductase;

DIA: Data-independent acquistion; F3H: Flavanone-3ß-hydroxylase; FDR: False discovery rate; FLS: Flavonol synthase; GO: Gene ontology; HSPs: Heat shock proteins; IAA: lodoacetamide; LHC: Light-harvesting chlorophyll; MAPK4: Mitogen-activated protein kinase 4; MDH: Malate dehydrogenase; MEP: Methylerythritol 4-phosphate; MVA: Mevalonic acid; MVK: Mevalonate kinase; PSI: Photosystem I; SAMMTases: S-adenosyl-L-methionine-dependent methyltransferases; SRG: Splicing-related gene; SWATH-MS: Sequential window acquisition of all theoretical spectra-mass spectrometry; TFA: Trifluoroacetic acid

Authors' contributions

CMX and SC designed and conducted the experiments, ZKL, SYC, TY, CX, LYG, YNH analyzed data, ZFY and ZJH drafted and revised the manuscript, ZFY and QSC conceived the project. All authors reviewed the results, revised the manuscript and approved its final version.

\section{Funding}

The work was supported by the National Natural Science Foundation of China (NSFC31701341), the NJFU project funding (GXL2018005), the China Postdoctoral Science Foundation (2017 M62280) and the Natural Science Foundation of Guangdong Province (2018A030313030).

Availability of data and materials

The data sets are included within the article and its Additional files.

Ethics approval and consent to participate

Not applicable.

Consent for publication

Not applicable.

Competing interests

The authors declare that they have no competing interests.

\section{Author details}

${ }^{1}$ Co-Innovation Center for Sustainable Forestry in Southern China, College of Biology and the Environment, Nanjing Forestry University, Nanjing 210037, Jiangsu Province, China. ${ }^{2}$ College of Horticulture, Nanjing Agricultural University, No. 1 Weigang, Nanjing 8210095, China. ${ }^{3}$ Medical Research Institute, Wuhan University and SpecAlly Life Technology Co., Ltd, Wuhan, China. ${ }^{4}$ State Key Laboratory of Crop Biology, College of Life Science, Shandong Agricultural University, Taian, Shandong, China. ${ }^{5}$ Southern Regional Collaborative Innovation Center for Grain and Oil Crops in China, Hunan Agricultural University, Changsha 410128, China. ${ }^{6}$ Department of Biology, Hong Kong Baptist University, and State Key Laboratory of Agrobiotechnology, The Chinese University of Hong Kong, Shatin, Hong Kong.

Received: 3 July 2019 Accepted: 5 September 2019

Published online: 24 October 2019

References

1. Khan MA, Olsen KM, Sovero V, Kushad MM, Korban SS. Fruit Quality Traits Have Played Critical Roles in Domestication of the Apple. Plant Genome. 2014;7(3):doi:https://doi.org/10.3835/plantgenome2014.04.0018. 
2. Marondedze $C$, Thomas LA. Apple hypanthium firmness. New insights from comparative proteomics. Appl Biochem Biotech. 2012;168(2):306-26.

3. Honda C, Kotoda N, Wada M, Kondo S, Kobayashi S, Soejima J, et al. Anthocyanin biosynthetic genes are coordinately expressed during red coloration in apple skin. Plant Physiol Bioch. 2002;40(11):955-62.

4. Koes R, Verweij W, Quattrocchio F. Flavonoids: a colorful model for the regulation and evolution of biochemical pathways. Trends Plant Sci. 2005; 10(5):236-42.

5. Lancaster JE, Dougall DK. Regulation of skin color in apples. Crit Rev Plant Sci. 1992:10(6):487-502.

6. Treutter D. Biosynthesis of phenolic compounds and its regulation in apple. Plant Growth Regul. 2001;34(1):71-89.

7. Lister CE, Lancaster JE, Walker JRL. Developmental changes in enzymes of flavonoid biosynthesis in the skins of red and green apple cultivars. Sci Food Agr. 2015;71(3):313-20.

8. Liu Y, Zhang $X$, Zhao Z. Effects of fruit bagging on anthocyanins, sugars, organic acids, and color properties of 'granny smith' and 'Golden delicious' during fruit maturation. Eur Food Res Technol. 2013;236(2):329-39.

9. Wang $\mathrm{H}$, Arakawa $\mathrm{O}$, Motomura $\mathrm{Y}$. Influence of maturity and bagging on the relationship between anthocyanin accumulation and phenylalanine ammonialyase (PAL) activity in 'Jonathan' apples. Postharvest Biol Tec. 2000;19(2):123-8.

10. Kano M, Takayanagi T, Harada K, Makino K, Ishikawa F. Antioxidative activity of anthocyanins from purple sweet potato, Ipomoera batatas cultivar Ayamurasaki. J Agric Chem Soc Japan. 2005;69(5):979-88.

11. Suda I, Oki T, Masuda M, Kobayashi M, Nishiba Y, Furuta S. Physiological functionality of purple-fleshed sweet potatoes containing anthocyanins and their utilization in foods. Jarq-Jpn Agr Res Q. 2003;37(3):167-73.

12. El-Sharkawy I, Liang D, Xu K. Transcriptome analysis of an apple (Malus $X$ domestica) yellow fruit somatic mutation identifies a gene network module highly associated with anthocyanin and epigenetic regulation. J Exp Bot. 2015;66(22):7359-76.

13. Gallardo K, Firnhaber C, Zuber H, Hericher D, Belghazi M, Henry C, et al. A combined proteome and transcriptome analysis of developing Medicago truncatula seeds. Mol Cell Proteomics. 2015;6(12):2165.

14. Zhu FY, Chen MX, Chan WL, Yang F, Tian Y, Song T, et al. SWATH-MS quantitative proteomic investigation of nitrogen starvation in Arabidopsis reveals new aspects of plant nitrogen stress responses. J Proteomics. 2018

15. Zhu FY, Chen MX, Su YW, Xu X, Ye NH, Cao YY, et al. SWATH-MS quantitative analysis of proteins in the Rice inferior and superior Spikelets during grain filling. Front Plant Sci. 2016;7(e0125934):1926.

16. Li S, Cao Q, Xiao W, Guo Y, Yang Y, Duan X, et al. Optimization of acquisition and data-processing parameters for improved proteomic quantification by sequential window Acquisition of all Theoretical Fragment ion Mass Spectrometry. J Proteome Res. 2017:16(2):738-47.

17. Allan A. C, Hellens, Roger P, Laing, William a. MYB transcription factors that colour our fruit. Trends Plant Sci. 2008;13(3):99-102.

18. Romo-Chacón A, Orozco-Avitia JA, Gardea AA, Guerrero-Prieto V, Soto-Parra JM. Hail net effect on photosynthetic rate and fruit color developent of 'Starkrimson' apple trees. J Amer Pomolog Soc. 2007:61 (4):174-8.

19. Schaart JG, Dubos C, Romero DLFI, van Houwelingen AM, de Vos RC, Jonker $\mathrm{HH}$, et al. Identification and characterization of MYB-bHLH-WD40 regulatory complexes controlling proanthocyanidin biosynthesis in strawberry (Fragaria X ananassa) fruits. New Phytol. 2013;197(2):454-67.

20. Yao P, Zhao H, Luo X, Gao F, Li C, Yao H, et al. Fagopyrum tataricum FtWD40 functions as a positive regulator of anthocyanin biosynthesis in transgenic tobacco. J Plant Growth Regul. 2017;36(3):755-65.

21. An X, Tian Y, Chen K, Wang X, Hao Y. The apple WD40 protein MdTTG1 interacts with bHLH but not MYB proteins to regulate anthocyanin accumulation. J Plant Physiol. 2012;169(7):710-7.

22. Liu Y, Hou H, Jiang X, Wang P, Dai X, Chen W, et al. A WD40 repeat protein from Camellia sinensis regulates anthocyanin and Proanthocyanidin accumulation through the formation of MYB-bHLH-WD40 ternary complexes. Int J Mol Sci. 2018;19(6):1686.

23. Xiu-Hong A, Yi T, Ke-Qin C, Xiao-Fei W, Yu-Jin H. The apple WD40 protein MdTTG1 interacts with bHLH but not MYB proteins to regulate anthocyanin accumulation. J Plant Physiol. 2012;169(7):710-7.

24. Banti V, Loreti E, Novi G, Santaniello A, Alpi A, Perata P. Heat acclimation and cross-tolerance against anoxia in Arabidopsis. Plant Cell Environ. 2010; 31(7):1029-37.

25. Gabai VL, Kabakov AE. Rise in heat-shock protein level confers tolerance to energy deprivation. FEBS Lett. 1993;327(3):247-50.
26. Downs CA, Heckathorn SA. The mitochondrial small heat-shock protein protects NADH:ubiquinone oxidoreductase of the electron transport chain during heat stress in plants. FEBS Lett. 1998;430(3):246.

27. Lüders J, Demand J, Höhfeld J. The ubiquitin-related BAG-1 provides a link between the molecular chaperones $\mathrm{Hsc70/Hsp70} \mathrm{and} \mathrm{the} \mathrm{proteasome.} \mathrm{J}$ Biol Chem. 2000;275(7):4613.

28. Ryhänen T, Hyttinen JMT, Kopitz J, Rilla K, Kuusisto E, Mannermaa E, et al. Crosstalk between $\mathrm{Hsp} 70$ molecular chaperone, lysosomes and proteasomes in autophagy-mediated proteolysis in human retinal pigment epithelial cells. Acta Ophthalmol. 2009;13(9b):3616-31.

29. Struck AW, Thompson ML, Wong LS, Micklefield J. S-adenosyl-methioninedependent methyltransferases: highly versatile enzymes in biocatalysis, biosynthesis and other biotechnological applications. Chembiochem. 2012; 13(18):2642-55.

30. Squarzoni P, Parveen F, Zanetti L, Ristoratore F, Spagnuolo A. FGF/ MAPK/Ets signaling renders pigment cell precursors competent to respond to Wnt signal by directly controlling ci-Tcf transcription. Development. 2011;138(7):1421-32.

31. Gutiérrez-Venegas $G$, Jiménez-Estrada M, Maldonado S. The effect of flavonoids on transduction mechanisms in lipopolysaccharide-treated human gingival fibroblasts. Int Immunopharmacol. 2007;7(9):1199-210.

32. Valbonesi $P$, Ricci L, Franzellitti S, Biondi C, Fabbri E. Effects of cadmium on MAPK signalling pathways and HSP70 expression in a human trophoblast cell line. Placenta. 2008;29(8):725-33.

33. Pulido P, Perello C, Rodriguezconcepcion M. New insights into plant isoprenoid metabolism. Mol Plant. 2012;5(5):964-7.

34. Meyer EH, Taylor NL, Millar AH. Resolving and identifying protein components of plant mitochondrial respiratory complexes using three dimensions of gel electrophoresis. J Proteome Res. 2008;7(2):786-94.

35. Tan YF, O'Toole N, Taylor NL, Millar AH. Divalent metal ions in plant mitochondria and their role in interactions with proteins and oxidative stress-induced damage to respiratory function. Plant Physiol. 2010; 152(2):747-61

36. Zhao W, Yang X, Yu H, Jiang W, Sun N, Liu X, et al. RNA-Seq-based transcriptome profiling of early nitrogen deficiency response in cucumber seedlings provides new insight into the putative nitrogen regulatory network. Plant Cell Physiol. 2014;pcu172.

37. Scheible WR, Morcuende R, Czechowski T, Fritz C, Osuna D, Palacios-Rojas N, et al. Genome-wide reprogramming of primary and secondary metabolism, protein synthesis, cellular growth processes, and the regulatory infrastructure of Arabidopsis in response to nitrogen. Plant Physiol. 2004; 136(1):2483-99

38. Galvez JH, Tai HH, Lague M, Zebarth BJ, Stromvik MV. The nitrogen responsive transcriptome in potato (Solanum tuberosum L.) reveals significant gene regulatory motifs. Sci Rep. 2016;6:26090.

39. He X, Ma H, Zhao X, Nie S, Li Y, Zhang Z, et al. Comparative RNA-sed analysis reveals that regulatory network of maize root development controls the expression of genes in response to $\mathrm{N}$ stress. PLoS One. 2016;11(3):e0151697.

40. Kim JY, Kim WY, Kwak KJ, Oh SH, Han YS, Kang H. Glycine-rich RNA-binding proteins are functionally conserved in Arabidopsis thaliana and Oryza sativa during cold adaptation process. J Exp Bot. 2010;61(9):2317-25.

41. Deng X, Lu T, Wang L, Gu L, Sun J, Kong X, et al. Recruitment of the NineTeen complex to the activated spliceosome requires AtPRMT5. Proc Natl Acad Sci U S A. 2016;113(19):5447-52.

42. Zhu FY, Chen MX, Ye NH, Shi L, Ma KL, Yang JF, et al. Proteogenomic analysis reveals alternative splicing and translation as part of the abscisic acid response in Arabidopsis seedlings. Plant J. 2017;91(3):518-33.

43. Cao S, Li J, Song S, Ran J, Xu G. AtGRP7 is involved in the regulation of abscisic acid and stress responses in arabidopsis. Cell Mol Biol Lett. 2006; 11(4):526-35.

44. Ciuzan O, Hancock J, Pamfil D, Wilson I, Ladomery M. The evolutionarily conserved multifunctional glycine-rich RNA-binding proteins play key roles in development and stress adaptation. Physiol Plantarum. 2014;153(1):1.

45. Kwak KJ, Kim YH. Characterization of transgenic Arabidopsis plants overexpressing GR-RBP4 under high salinity, dehydration, or cold stress. J Exp Bot. 2005;56(421):3007-16.

46. Staiger D. RNA-binding proteins and circadian rhythms in Arabidopsis thaliana. Philos Trans R Soc Lond Ser B Biol Sci. 2001;356(1415):1755-9.

47. Schmidt F, Marnef A, Cheung M, Wilson ID, Hancock JT, Staiger D, et al. A proteomic analysis of oligo (dT)-bound mRNP containing oxidative stress- 
induced Arabidopsis thaliana RNA-binding proteins ATGRP7 and ATGRP8. Mol Biol Rep. 2010;37(2):839-45.

48. Lorkoviäł ZJ, Wieczorek Kirk DA, Klahre U, Hemmings-Mieszczak M, Filipowicz W. RBP45 and RBP47, two oligouridylate-specific hnRNP-like proteins interacting with poly(a)+ RNA in nuclei of plant cells. RNA. 2000; 6(11):1610-24.

49. Palanivelu R, Belostotsky DA, Meagher RB. Arabidopsis thaliana poly (a) binding protein 2 (PAB2) functions in yeast translational and mRNA decay processes. Plant J. 2010;22(3):187-98.

50. Wu WW, Wang G, Baek SJ, Shen R. Comparative study of three proteomic quantitative methods, DIGE, CICAT, and iTRAQ, using 2D gel- or LC-MALDI TOF/TOF. J Proteome Res. 2006;5(3):651-8.

51. Zhu FY, Chan WL, Chen MX, Kong RP, Cai C, Wang Q, et al. SWATH-MS quantitative proteomic investigation reveals a role of Jasmonic acid during Lead response in Arabidopsis. J Proteome Res. 2016;15(10):3528.

52. Komor MA, Hiemstra AC, Pham TV, Piersma SR, Sebra RP, Han BW, et al. Proteogenomic analysis of alternative splicing: the search for novel biomarkers for colorectal cancer. Cancer Res. 2016;76(14 Supplement):848.

53. Kumar D, Yadav AK, Jia X, Mulvenna J, Dash D. Integrated transcriptomicproteomic analysis using a proteogenomic workflow refines rat genome annotation. Mol Cell Proteomics. 2016;15(1):329-39.

54. Walker AR, Lee E, Robinson SP. Two new grape cultivars, bud sports of cabernet sauvignon bearing pale-coloured berries, are the result of deletion of two regulatory genes of the berry colour locus. Plant Mol Biol. 2006;62:623-35.

55. Mullins MG. Changes in anthocyanin and Phenolics content of grapevine leaf and fruit tissues treated with sucrose, nitrate, and abscisic acid. Plant Physiol. 1976;58(4):468-72.

56. Xu Y, Feng S, Jiao Q, Liu C, Zhang W, Chen W, et al. Comparison of MdMYB1 sequences and expression of anthocyanin biosynthetic and regulatory genes between Malus domestica Borkh. Cultivar 'Ralls' and its blushed sport. Euphytica. 2012;185(2):157-70.

57. Lei Z, Xu Y, Yue J, Wang J, Yue Y, Yang Y, et al. Effect of floral cluster pruning on anthocyanin levels and anthocyanain-related gene expression in 'Houman' grape. Hortic Res. 2016;3:16037.

58. Haverland NA, Fox HS, Ciborowski P. Quantitative proteomics by SWATH-MS reveals altered expression of nucleic acid binding and regulatory proteins in HIV-1-infected macrophages. J Proteome Res. 2014;13(4):2109-19.

59. Cheadle C, Vawter MP, Freed WJ, Becker KG. Analysis of microarray data using Z score transformation. J Mol Diagn. 2003;5(2):73-81.

\section{Publisher's Note}

Springer Nature remains neutral with regard to jurisdictional claims in published maps and institutional affiliations.

Ready to submit your research? Choose BMC and benefit from:

- fast, convenient online submission

- thorough peer review by experienced researchers in your field

- rapid publication on acceptance

- support for research data, including large and complex data types

- gold Open Access which fosters wider collaboration and increased citations

- maximum visibility for your research: over $100 \mathrm{M}$ website views per year

At $\mathrm{BMC}$, research is always in progress.

Learn more biomedcentral.com/submissions 